05,13

\title{
Трехмерное моделирование нелинейной динамики доменных границ в пленках с перпендикулярной анизотропией
}

\author{
(C) В.В. Зверев ${ }^{1}$, Б.Н. Филиппов ${ }^{1,2}$, М.Н. Дубовик ${ }^{1,2}$ \\ ${ }^{1}$ Уральский федеральный университет, \\ Екатеринбург, Россия \\ ${ }^{2}$ Институт фризики металлов УрО РАН, \\ Екатеринбург, Россия \\ E-mail: vvzverev49@gmail.com
}

(Поступила в Редакцию 27 июля 2016 г.)

\begin{abstract}
На основе численного решения уравнений Ландау-Лифшица-Гильберта выполнено трехмерное компьютерное моделирование статических конфигураций намагниченности и динамических процессов в доменной границе магнитно-одноосной пленки с перпендикулярной анизотропией. Рассчитанные статические состояния соответствуют доменной границе, содержащей блоховские линии с поверхностным распределением намагниченности, зависящим от толщины пленки. Показано, что такие структуры могут быть охарактеризованы определенными значениями гомотопического индекса. Установлено, что существующие в объеме пленки вихревые и антивихревые структуры образуют вихревые нити. Найден способ визуализации совместного движения вихревых нитей и блоховских точек, основанный на численном расчете значений гомотопического индекса и числа вращений.

Работа выполнена в рамках государственного задания ФАНО России (тема „Магнит“, № 01201463328) при частичной поддержке УрО РАН (проект № 15-9-2-33), финансовой поддержке согласно постановлению № 211 Правительства Российской федерации, контракт № 02.А03.21.0006 и финансовой поддержке Министерства образования и науки РФ на основе госзадания, проект № 2705.
\end{abstract}

DOI: 10.21883/FTT.2017.03.44161.310

\section{1. Введение}

Открытие доменных границ (ДГ) и их подвижности явилось фундаментальным вкладом в представления о процессах намагничивания и перемагничивания в ферромагнетиках [1], сыграв ключевую роль в понимании многих практически важных свойств магнитных веществ и обеспечив возможность получения материалов с необходимыми свойствами. Начавшееся достаточно давно изучение статических и динамических свойств ДГ активно продолжается и в настоящее время. Наряду с традиционной задачей изучения полезных свойств магнитных материалов появились новые задачи, связанные с использованием ДГ и элементов, составляющих их структуру, в современных высокоемких и высокоскоростных энергонезависимых устройствах записи информации с произвольной выборкой [2,3], элементах логики [4] и спинтронных устройствах [5-8].

С момента появления первой работы Ландау и Лифшица [9] сами представления о структуре доменных стенок изменились до неузнаваемости. Оказалось, что в пленках, широко используемых на практике, ДГ не являются одномерными и могут обладать симметричной или асимметричной вихреподобной структурой [10,11]. Кроме того, эти стенки могут включать в себя различные структурные элементы, среди которых принято выделять вертикальные и горизонтальные блоховские линии (ВБЛ и ГБЛ) и сингулярные (блоховские) точки (СТ) (см., например [12-14]). Изучение динамики намагниченности в пленках показало, что в статических внешних магнитных полях или в присутствии спинполяризованного тока может не только возникать движение ДГ как целого, но и происходить перестройка ее внутренней структуры. Правильное понимание общей картины динамического поведения намагниченности является важным при разработке устройств записи и считывания информации, основанных на движении ДГ.

В настоящее время интенсивно развивается моделирование статических и динамических свойств спиновых структур, учитывающее локальные трехмерные спиновые образования, например, новые типы блоховских линий [15-20], локализованных на вихреподобных асимметричных стенках в пленках с плоскостной анизотропией. Обнаружено, что нестационарное движение ДГ сопровождается сложным движением вихрей, антивихрей и СТ, их рождением и аннигиляцией. Изучение этих процессов имеет не только прикладное, но и фундаментальное значение, поскольку позволяет обнаруживать закономерности, справедливые и для других нелинейных сред.

В настоящей работе на основе микромагнитного подхода выполняется трехмерное компьютерное моделирование динамических процессов, происходящих при движении доменных стенок в магнитных пленках с перпендикулярной анизотропией. Такие пленки, обладающие как полупроводниковыми, так и магнитными свойствами, считаются перспективными для применения в спинтронных устройствах, что интересно с точки зрения развития информационных технологий. Примером 

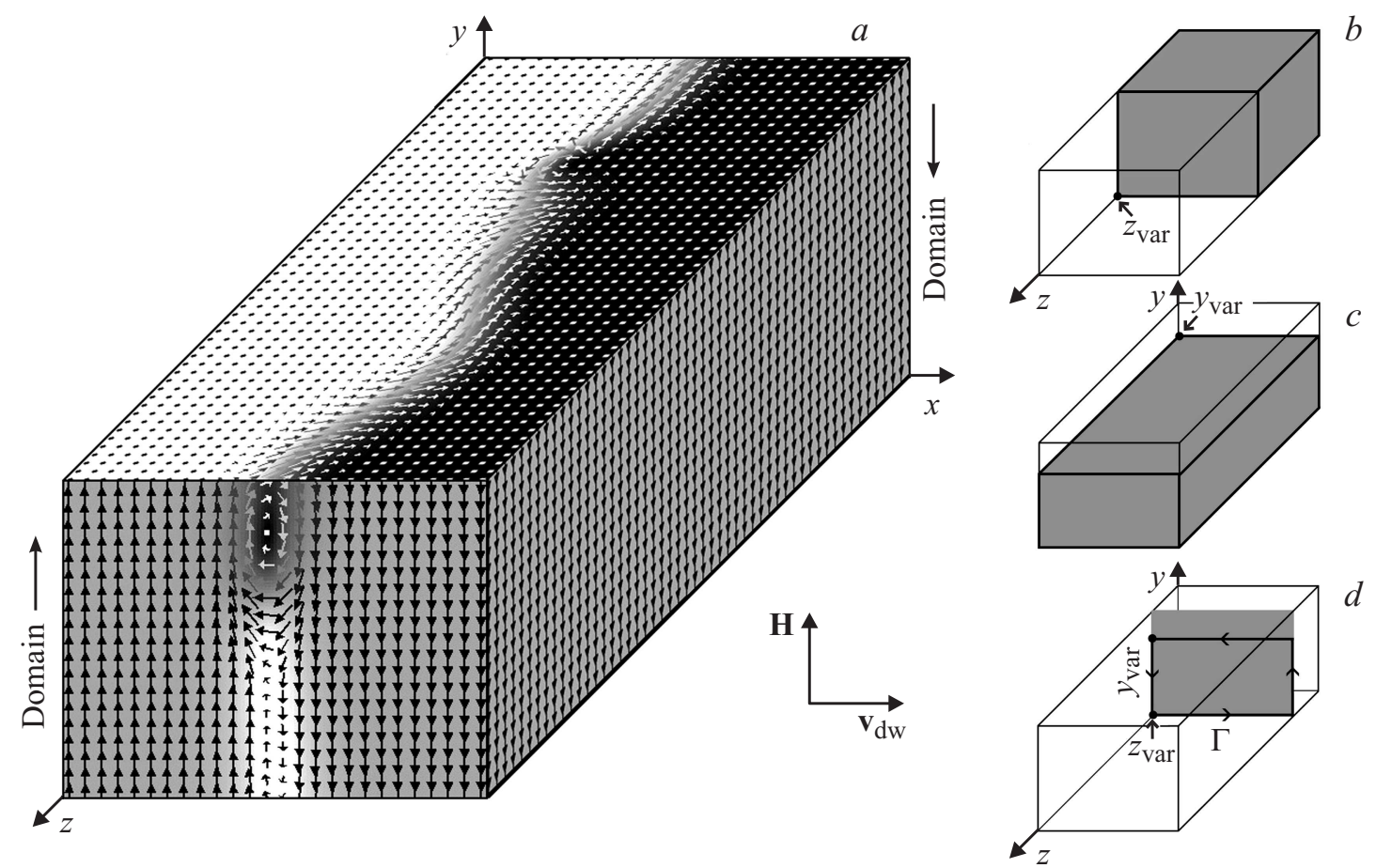

Рис. 1. Геометрия расчетной области и пример распределения намагниченности в ДГ, движущейся в направлении $\mathbf{v}_{\mathrm{dw}}$ под действием магнитного поля Н $(a)$, граничные поверхности параллелепипедов, используемых при расчете зависимостей гомотопического индекса $\chi$ от величин $z_{\text {var }}(b)$ и $y_{\text {var }}(c)$, задающих положения подвижных граней, и прямоугольный контур $Г$, с помощью которого рассчитывается зависимость числа вращения $\gamma$ от длины стороны прямоугольника $y_{\text {var }}$ и положения содержащей контур плоскости $z=z_{\text {var }}(d)$.

могут служить пленки из GaMnAs, получаемые специальным образом при некоторой рассогласованности параметров кристаллических решеток пленки и подложки (см., например, $[8,12]$ ).

Мы опираемся на результаты работ [18-20], в которых было выполнено трехмерное моделирование различных динамических процессов в магнитноодноосных пленках с плоскостной анизотропией. В частности, используется разработанный в [18-20] метод визуализации топологической структуры распределений намагниченности, основанный на численном определении значений „топологических зарядов“ двух типов. Этот метод позволяет наблюдать за движением вихрей, антивихрей и СТ как в глубине пленки, так и на ее поверхности. В [20] было установлено, что вихревые или антивихревые распределения намагниченности, формирующиеся на поверхности пленки с плоскостной анизотропией и в различных ее сечениях, связаны и образуют единую топологическую структуру. При описании такой структуры удобно использовать понятие „вихревая нить“, которую можно представить в виде непрерывной пространственной кривой, соединяющей центры ко́ров вихрей/антивихрей, локализованных в параллельных и ориентированных определенным образом плоскостях. Вихревые нити могут выходить на поверхность пленки в области ВБЛ, либо образовывать внутренние кольцевые структуры. В частности, асимметричная вихревая ДГ [10] без ВБЛ содержит вихревую нить, тянущуюся параллельно поверхностям магнитной пленки и не выходящую на поверхность. В данной работе показано, что концепция вихревых нитей может быть с успехом применена и к случаю пленок с перпендикулярной анизотропией.

\section{2. Постановка задачи}

Проводится компьютерное моделирование статики и динамики распределения намагниченности в одноосной магнитной пленке. Полагаем, что ось $z$ направлена вдоль ДГ, а ось $y$ - вдоль оси легкого намагничивания (ОЛН) перпендикулярно к поверхности пленки. В качестве расчетной области выбран прямоугольный параллелепипед, имеющий следующие размеры по координатным осям: $l_{x}$ - расстояние между доменами, $l_{y}$ - толщина пленки, $l_{z}$ - длина отрезка ДГ (рис. $1, a$ ).

Будем использовать для описания динамики намагниченности уравнение Ландау-Лифшица-Гильберта

$$
\frac{d \mathbf{m}}{d t}=-|\gamma|\left[\mathbf{m} \times \mathbf{H}_{\mathrm{eff}}\right]+\alpha\left[\mathbf{m} \times \frac{d \mathbf{m}}{d t}\right],
$$

записанное для поля направлений $\mathbf{m}=\mathbf{M} / M_{s}$ вектора намагниченности $\mathbf{M}$; здесь $M_{s}-$ намагниченность насыщения $\left(\mathbf{M}^{2}=M_{s}^{2}\right)$. В правую часть уравнения (1) входят эффективное магнитное поле $\mathbf{H}_{\mathrm{eff}}=-\delta W / \delta \mathbf{M}$ $=\mathbf{H}_{e}+\mathbf{H}_{a}+\mathbf{H}^{(m)}+\mathbf{H}$, равное функциональной производной энергии магнетика $W$ и являющееся суммой 


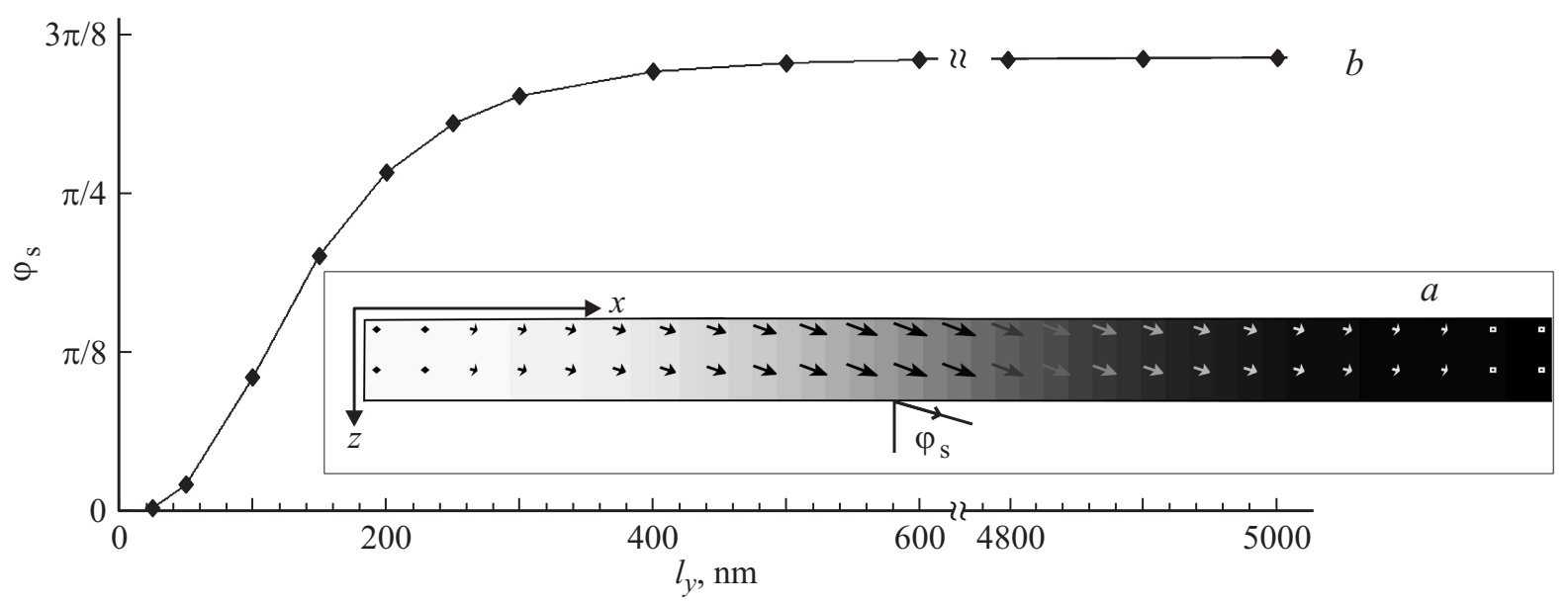

Рис. 2. Угол $\varphi_{S}$ и его зависимость от толщины пленки.

обменного поля $\mathbf{H}_{e}=2 A M_{s}^{-1} \Delta \mathbf{m}$, поля магнитной анизотропии $\mathbf{H}_{a}=2 K M_{s}^{-1} \mathbf{j}(\mathbf{j m})$, магнитостатического поля $\mathbf{H}^{(m)}$ и постоянного однородного поля $\mathbf{H}=\mathbf{j} H ; \mathbf{a}$ также гиромагнитное отношение $\gamma$, параметр затухания Гильберта $\alpha$, обменный параметр $A$ и константа анизотропии $K$ (здесь $\mathbf{j}$ - орт оси $y$ ). Задавая специально подобранные кусочнопостоянные распределения намагниченности и находя установившиеся решения уравнения (1) при $H=0$, мы получаем различные статические конфигурации намагниченности и используем их в качестве начальных условий при моделировании динамики. Те же статические решения могут быть найдены путем минимизации полной энергии магнетика $W$. Зададим условия на границах доменов

$$
\left.\mathbf{m}\right|_{x=0}=\mathbf{j},\left.\quad \mathbf{m}\right|_{x=l_{x}}=-\mathbf{j},
$$

а также циклические граничные условия

$$
\left.\mathbf{m}\right|_{z=0}=\left.\mathbf{m}\right|_{z=l_{z}} .
$$

Последние позволяют не рассматривать эффекты, связанные с обрыванием намагниченности на концах отрезка ДГ. Условие (3) можно интерпретировать как существование закольцованного отрезка ДГ; проходя вдоль $z$ от точки $z=0$ до точки $z=l_{z}$, мы завершаем однократный цикл обхода по такому отрезку. Будем также считать, что на граничных поверхностях пленки $y=0, y=l_{y}$ намагниченность незакреплена [22].

При выполнении расчетов использовалась программа для проведения микромагнитного моделирования mumax3, разработанная в исследовательской группе DyNaMat (Гентский университет, Бельгия) [23]. Рассматривалась пленка с параметрами $A=10^{-11} \mathrm{~J} / \mathrm{m}$, $K=2.5 \cdot 10^{4} \mathrm{~J} / \mathrm{m}^{3}, M_{s}=100 \mathrm{G}, \alpha=0.2$ (наличие ЦМДматериалов с близкими параметрами отмечалось, к примеру, в обзоре [24]). При моделировании движения ДГ, помещенной в постоянное магнитное поле, был использован метод компенсации смещения ДГ, позволяющий расчетной области следовать за движущейся ДГ.
Распределения намагниченности, получающиеся путем численного моделирования, могут содержать СТ, вблизи которых нарушаются условия континуального подхода. В работе [25] было показано, что, хотя в этом случае условия использования микромагнитного подхода оказываются нарушенными, в целом результаты расчета остаются корректными, поскольку вклад от СТ в полную энергию системы мал. Рассматривая распределения с СТ как физически допустимые, мы опираемся на этот результат.

Здесь и ниже будем для краткости называть симметричную вихревую („скрученную“) ДГ с отсутствующими в ней блоховскими линиями и точками однородной. Для уточнения зависимости параметров однородной ДГ от толщины пленки рассчитывались статические конфигурации при $l_{x}=400 \mathrm{~nm}, l_{y}=25-5000 \mathrm{~nm}, l_{z}=12.5 \mathrm{~nm}$ (здесь и далее шаг сетки $3.125 \mathrm{~nm}$ ). Во всем диапазоне толщин ДГ имеет в сечениях $z=$ const симметричную вихревую структуру. В точках, лежащих на граничных поверхностях пленки $\left(y=0, y=l_{y}\right)$ и принадлежащих плоскости осевого сечения ДГ, перпендикулярной этим поверхностям, намагниченность образует с осью $z$ угол $\varphi_{S}$ (см. рис. 2,a). Будучи близким к нулю для тонких пленок, с ростом толщины пленки этот угол растет (рис. 2,b), стремясь к некоторому предельному значению. Данная закономерность согласуется с результатами работы [26]. Согласно результатам расчетов, это предельное значение стремится к $\pi / 2$ при уменьшении величины отношения $A / M_{s}^{2}$. Значения угла $\varphi_{S}$ существенным образом влияют на распределение намагниченности в ВБЛ (см. ниже).

\section{3. Структура неподвижной ДГ, содержащей ВБЛ}

Для изучения структуры ВБЛ были рассчитаны статические конфигурации при $l_{x}=400 \mathrm{~nm}, l_{y}=100$ и $300 \mathrm{~nm}, l_{z}=800 \mathrm{~nm}$. Как следует из рис. 2, при меньшем значении толщины угол $\varphi_{S}$ мал, а при большем 

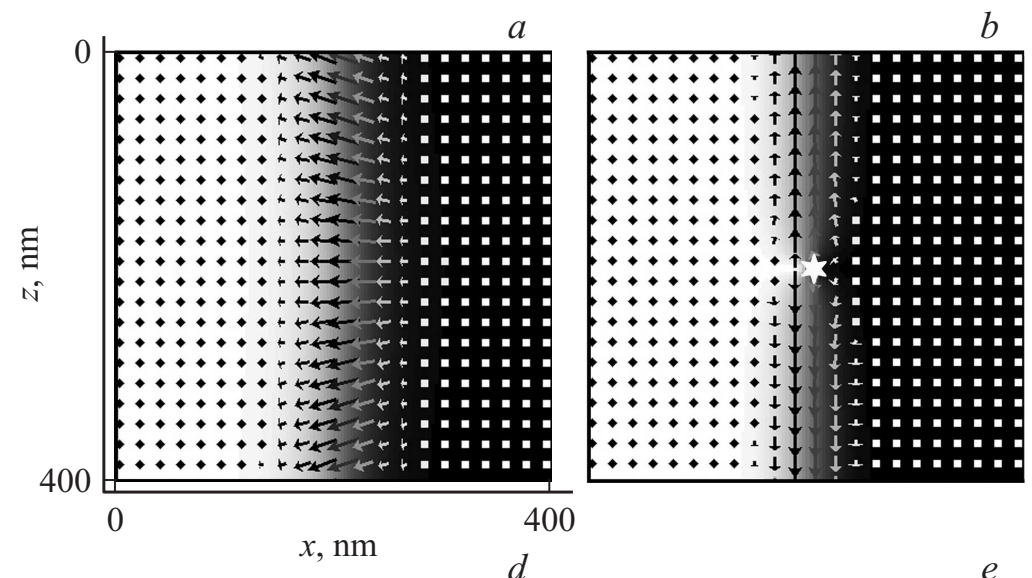

$b$

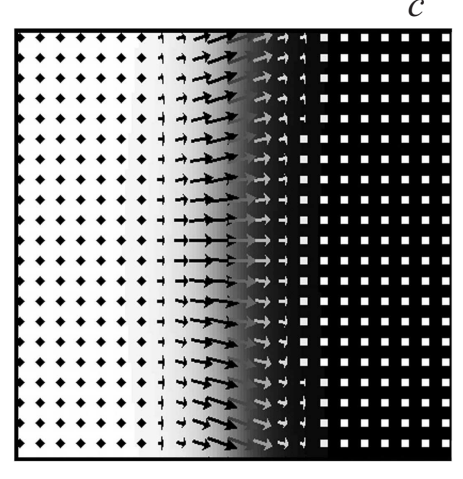

$d$
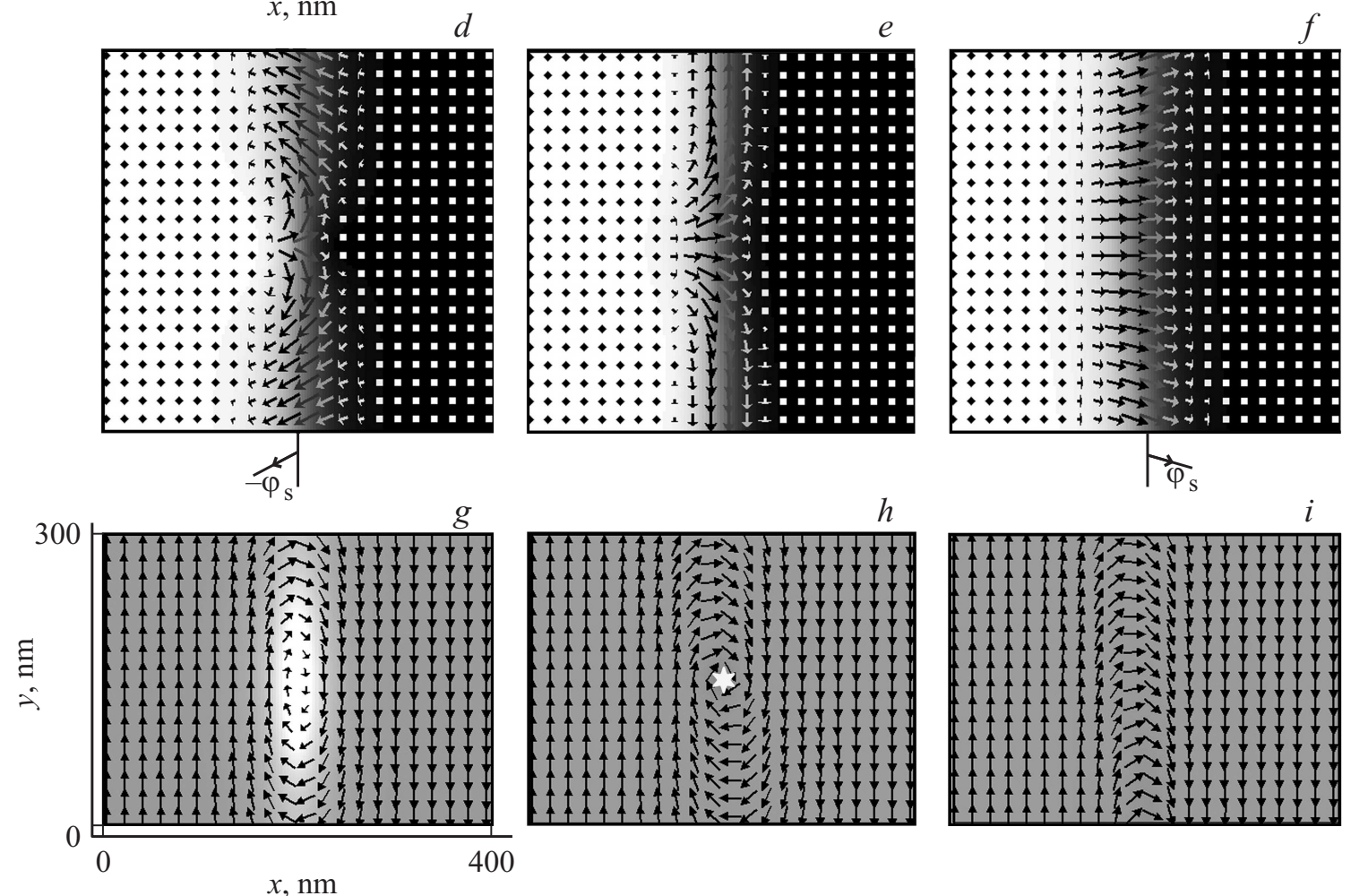

Рис. 3. Распределения намагниченности в области ВБЛ на нижней $(a, d)$ и верхней $(c, f)$ границах пленки, а также в среднем сечении, параллельном границам $(b, e)$. Рисунки относятся к ВБЛ, содержащей СТ $(a-c)$ и не содержащей СТ $(d-f)$. Внизу представлены распределения намагниченности в сечении плоскостью, перпендикулярной ДГ и граничным поверхностям пленки, в случае отсутствия ВБЛ $(g)$, а также если сечение проходит через СТ $(h)$ или через центральную часть ВБЛ без СТ $(i)$. Положение СТ отмечено звездочкой.

близок к предельному значению. Таким образом, мы получаем возможность сравнить и проанализировать две качественно различающиеся конфигурации. При уменьшении толщины $\varphi_{S} \rightarrow 0$ и распределение намагниченности становится близким к одномерному (направление $\mathbf{m}$ зависит только от координаты $x$ ).

При наложении периодического граничного условия (3) отрезок ДГ должен содержать не менее двух ВБЛ. Здесь мы рассмотрим ВБЛ двух типов; ВБЛ типа I содержит СТ, ВБЛ типа II на границах пленки имеет участки с закрученной намагниченностью. Изображения распределений намагниченности в области ВБЛ на границах пленки и в различных сечениях представлены на рис. 3. Оба типа ВБЛ ранее обсуждались в литературе по ЦМД.

Возможны четыре различных типа распределения намагниченности для закольцованного отрезка ДГ с двумя ВБЛ (рис. 4,5). В случае $A$ обе ВБЛ имеют тип I; в случае $B$ ДГ содержит ВБЛ типа I и II; в случаях $C$ и $D$ обе ВБЛ имеют тип II. В последних двух случаях существенную роль играет то, что одну ВБЛ типа II можно расположить относительно другой ВБЛ того же типа двумя неэквивалентными способами. Если $\varphi_{S} \neq 0$, конфигурация намагниченности в ВБЛ типа II не является зеркально-симметричной относительно плоскости $y=\frac{1}{2} l_{y}$. Смещаясь вдоль осевой линии ДГ из точки 


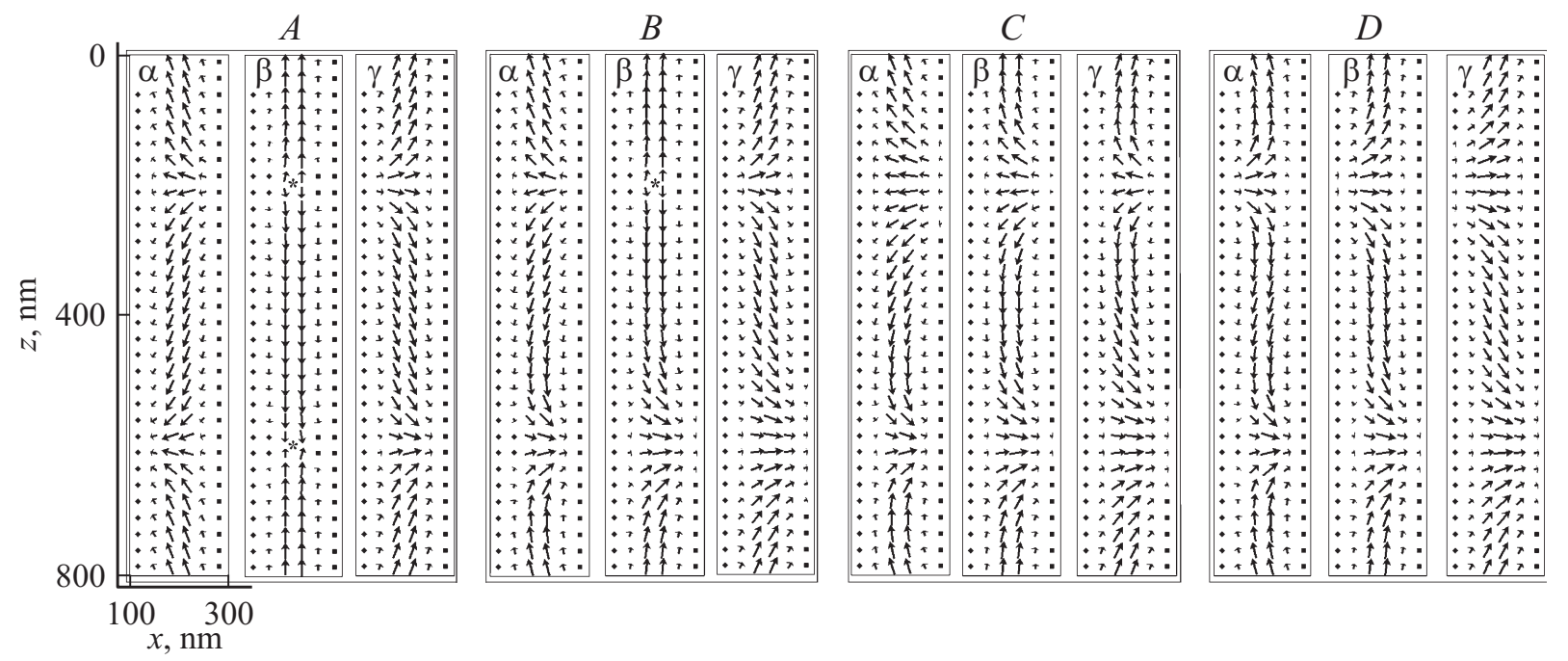

Рис. 4. Распределения намагниченности в области ДГ на нижней $(\alpha)$ и верхней $(\gamma)$ границах пленки толщиной $100 \mathrm{~nm}$, а также в среднем сечении, параллельном граничным поверхностям этой пленки $(\beta)$. Представлены четыре возможные конфигурации, каждая из которых содержит две ВБЛ, относящиеся к одному из двух типов. На ДГ наложено циклическое граничное условие. Звездочками отмечены положения СТ.
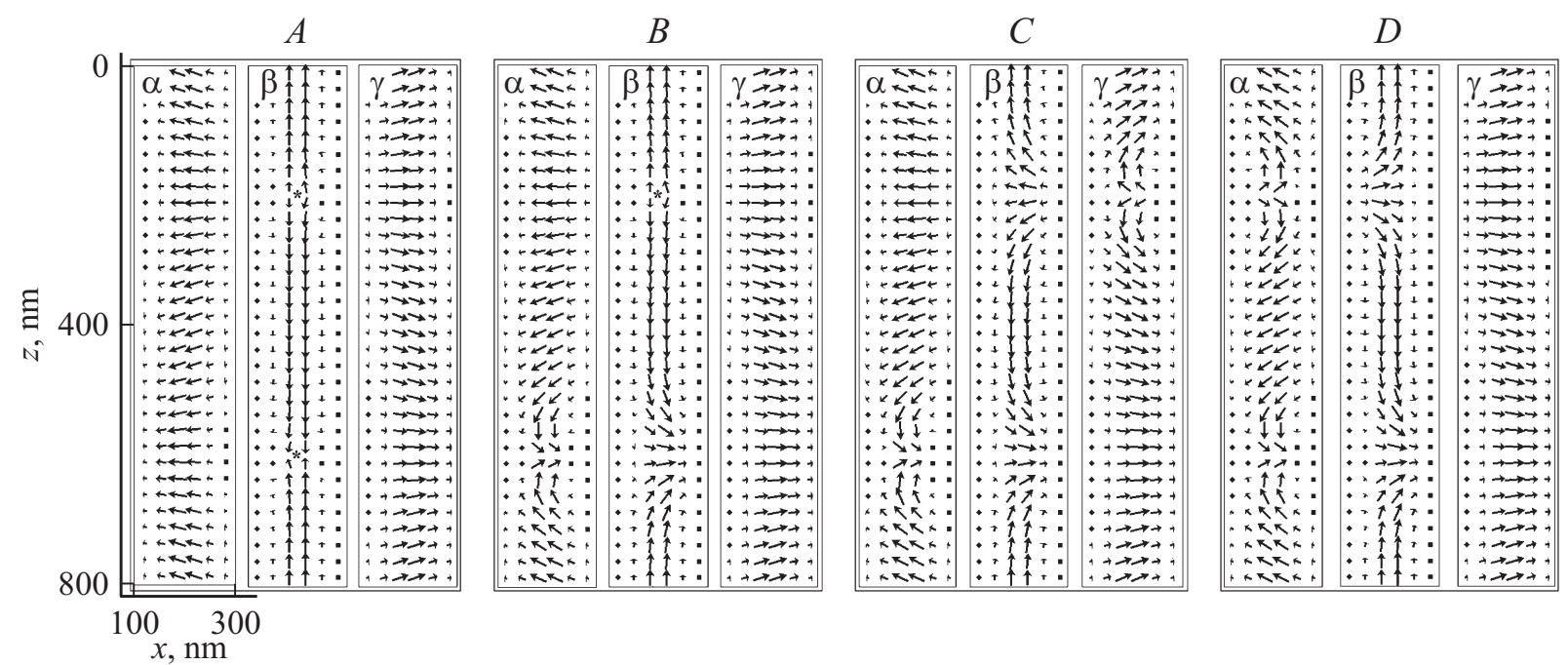

Рис. 5. Распределения намагниченности, аналогичные изображенным на предыдущем рисунке, для пленки толщиной $300 \mathrm{~nm}$.

$z=0$ в точку $z=l_{z}$ (что соответствует, с учетом условия периодичности (3), перемещению на один период), мы видим, что в случаях $A$ и $D$ угол полного поворота вектора намагниченности на верхней и нижней границах пленки равен нулю. Это означает, что, не производя разрывов, конфигурации намагниченностей в этих случаях можно превратить в поле векторов намагниченности отрезка однородной ДГ (в случае $A$ должна произойти аннигиляция двух СТ). При таком же однократном обходе цикла в случае В на нижней границе (рис. 4 и $5, B \alpha$ ), а в случае $C$ на верхней и нижней границах пленки (рис. 4 и $5, C \alpha$ и $C \gamma$ ) суммарный угол поворота равен $2 \pi$. В этом случае распределение намагниченности в закольцованном отрезке ДГ является „закрученным“ и топологически не эквивалентно распределению в отрезке однородной ДГ. Если $\varphi_{S} \approx 0$ (рис. 4), при прохождении области локализации ВБЛ в процессе движения вдоль ДГ, в случаях ВБЛ как типа I, так и типа II, углы поворота векторов поверхностной намагниченности близки к $\pi$. При $\varphi_{S} \approx \pi / 2$ (рис. 5) для ВБЛ типа I углы поворота близки к нулю; для ВБЛ типа II угол поворота на одной из граничных поверхностей мал, на другой же близок к $2 \pi$.

Выполненные нами расчеты статических конфигураций ДГ с ВБЛ уточняют данные, полученные ранее (структура ВБЛ рассчитывалась, к примеру, в работах [27-32]). В частности, если в пленках больших толщин $\left(\varphi_{S} \approx \pi / 2\right)$ ВБЛ типа II хорошо различима только на одной поверхности пленки (этот случай и рассматривался в [27-32]), то при уменьшении $l_{y}$ об- 
ласть локализации ВБЛ становится хорошо заметна на обеих поверхностях. Отметим также, что результаты моделирования статических конфигураций, полученные с достаточно высокой степенью точности, ценны для нас тем, что являются основой для моделирования динамики ДГ с учетом изменения их внутренней структуры.

В работе [20] был проведен анализ статических конфигураций намагниченности и динамических перестроек этих конфигураций в магнитных пленках с плоскостной анизотропией, основанный на использовании гомотопического индекса. Эта величина может быть определена выражением $\chi=(4 \pi)^{-1} \int d \Omega(\mathbf{r})$ и имеет простой геометрический смысл: она равна суммарному телесному углу, который пробегает вектор поля намагниченности $\mathbf{M}(\mathbf{r})$ в том случае, если радиус-вектор $\mathbf{r}$ пробегает некоторую область в координатном пространстве. Если областью интегрирования является замкнутая поверхность, индекс $\chi$ равен алгебраической сумме вкладов от CТ, имеющихся в объеме, ограниченном этой поверхностью, при том, что одиночная СТ дает вклад, равный \pm 1 [12,33-35] (индекс $\chi$ в этом случае часто называют топологическим зарядом). В тех случаях, когда интегрирование ведется по поверхности, гомотопический индекс обычно записывается в виде

$$
\chi=\frac{1}{4 \pi} \iint_{S} \mathbf{g} d \mathbf{s},
$$

где $\mathbf{g}=[\nabla \cos \Theta \times \nabla \Phi]-$ плотность гиротропного вектора, выраженная через углы, определяющие положения вектора намагниченности: $\mathbf{m}=(\sin \Theta \cos \Phi, \sin \Theta \sin \Phi, \cos \Theta)$.

Индекс $\chi$ используется при анализе топологических структур на граничных поверхностях трехмерных образцов, которые аналогичны структурам в двумерном ферромагнетике [36]. Можно показать [37,38], что поверхностные вихри и антивихри дают в значение $\chi$ полуцелые вклады, которые описываются выражением $\chi=-\lambda q / 2$, где $q=1$ для вихря, $q=-1$ для антивихря; значение множителя $\lambda= \pm 1$ определяется ориентацией вектора намагниченности в центре вихря (антивихря) относительно нормали к граничной поверхности. В работе [20] было показано, что при вычислении интеграла (4) по различным замкнутым поверхностям результат зависит только от того, сколько СТ охвачено этими поверхностями; суммарный вклад, который вносят в значение $\chi$ вихри и антивихри, всегда равен нулю. Это означает, что изменение числа поверхностных вихрей (антивихрей), дающих такие вклады, происходит согласованным образом, что свидетельствует о наличии во внутренней области образца вихревых нитей (см. раздел 1). Заметим, что интересная задача визуализации нитей, фактически поставленная в работе [20], не была в этой работе решена. Ниже, в разделе 4, мы приведем результаты динамической визуализации вихревых нитей в рассматриваемых здесь пленках.

Распространим подход, предложенный в [20], на случай магнитной пленки с перпендикулярной анизотро- пией. Используя данные о распределениях намагниченности, полученные численно, будем с помощью формулы (4) рассчитывать значения индекса $\chi$ при различных $z_{\text {var }}$, выбирая в качестве поверхностей интегрирования грани параллелепипеда, изображенного на рис. $1, b$. Здесь и ниже $z_{\text {var }}$ и $y_{\text {var }}$ описывают положение подвижных граней параллелепипедов. Графики зависимостей от $z=z_{\text {var }}$ вкладов верхней грани $\chi_{t}$, нижней грани $\chi_{b}$, а также разности вкладов фронтальной и тыльной граней $\chi_{f}-\chi_{r}$, для случая $B$ и пленок толщиной $100 \mathrm{~nm}$ и $300 \mathrm{~nm}$, изображены на рис. 6 (вклады боковых граней равны нулю). Мы видим, что так же, как и в случаях, рассмотренных в работе [20], области локализации ВБЛ на ДГ (теперь это не вихри и антивихри, а области разворота намагниченности) дают вклады в значения $\chi_{t}$ и $\chi_{b}$. Соответствующие им плавные „скачки“ изображены на рис. $6, a, b, e, f$. Теперь амплитуды этих скачков не являются, однако, целыми или полуцелыми, а зависят от толщины пленки. На графике для $\chi_{f}-\chi_{r}$ (наряду с единичным скачком, связанным с наличием СТ) также видны вклады от ВБЛ. На графиках для суммарных величин $\chi_{\Sigma}=\chi_{t}+\chi_{b}+\chi_{f}+\chi_{r}($ рис. $6, d, h)$ имеется, однако, только вклад от СТ, вклады же от ВБЛ отсутствуют. Это позволяет предположить, что вихревые нити существуют и в пленках с перпендикулярной анизотропией.

Рассмотрим простую приближенную теорию, позволяющую объяснить зависимость от $\varphi_{S}$ амплитуд скачков, имеющихся на графиках $a, b, e, f$ на рис. 6. Ограничимся частным случаем ВБЛ типа II, предполагая, что она находится в параллелепипеде $G=\left\{0<x<l_{x}, 0<y<l_{y}, 0<z<\frac{1}{2} l_{z}\right\}$ (соответствующие распределения намагниченности на границах пленки и в различных сечениях изображены на рис. $3, d, e, f, g, i)$. Введем модельные функции, чтобы описать распределения намагниченности на верхней и нижней гранях

$$
\begin{gathered}
m_{x}=\sin \Theta(x) \sin \Phi(z), m_{y}=\cos \Theta(x), \\
m_{z}=\sin \Theta(x) \cos \Phi(z) .
\end{gathered}
$$

на фронтальной и тыльной гранях

$$
\begin{gathered}
m_{x}=\sin \Theta(x) \sin \Psi(y), m_{y}=\cos \Theta(x), \\
m_{z}=\sin \Theta(x) \cos \Psi(y) .
\end{gathered}
$$

Чтобы получить качественно верные распределения на ребрах параллелепипеда, следует наложить на функции, входящие в (5), (6), граничные условия: на всех гранях (кроме боковых)

$$
\Theta(0)=0, \quad \Theta\left(\frac{1}{2} l_{x}\right)=\pi / 2, \quad \Theta\left(l_{x}\right)=\pi,
$$

на верхней грани

$$
\Phi(0)=\pi-\varphi_{S}, \quad \Phi\left(\frac{1}{4} l_{z}\right)=\pi / 2, \quad \Phi\left(\frac{1}{2} l_{z}\right)=\varphi_{S},
$$



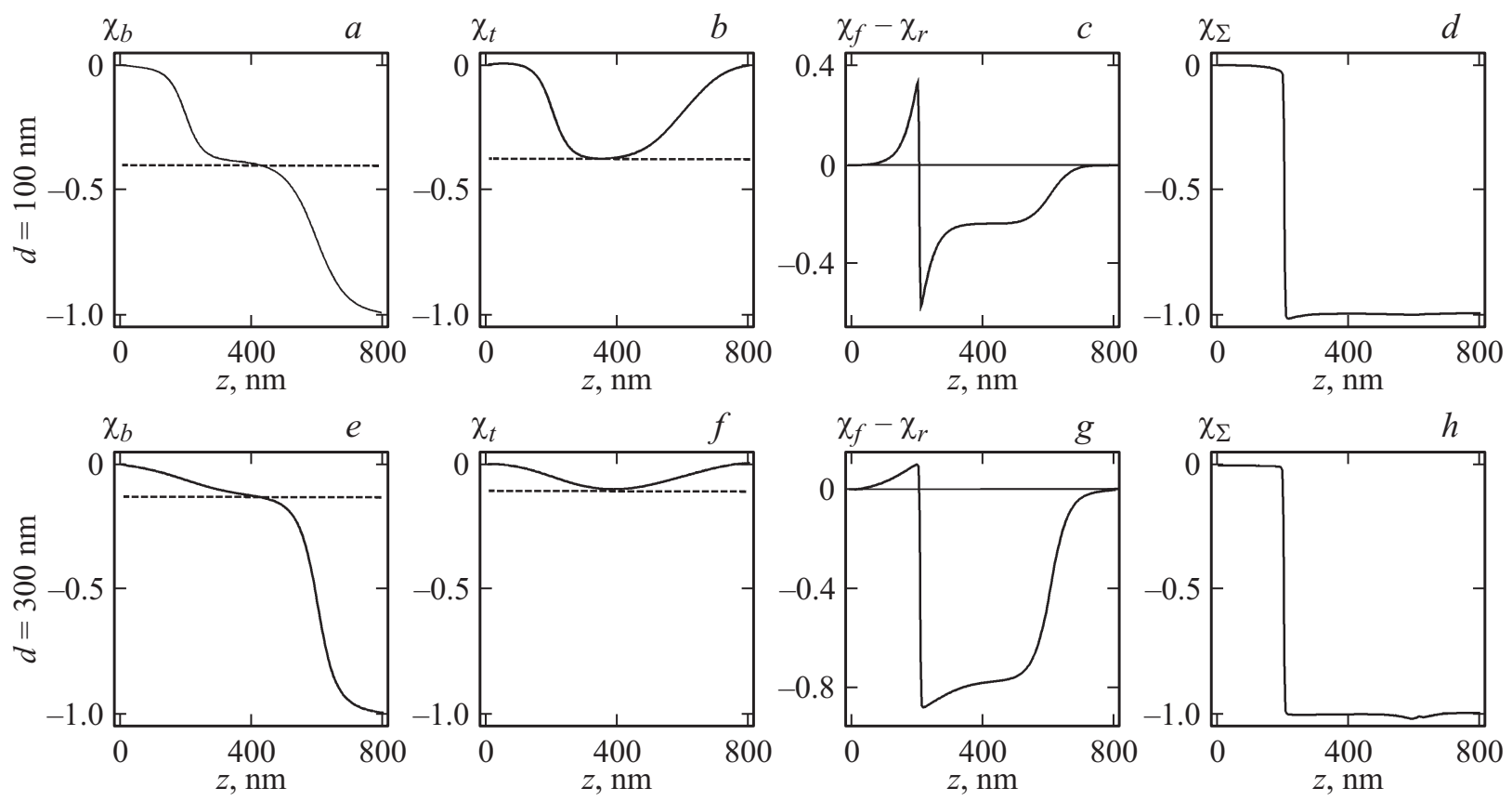

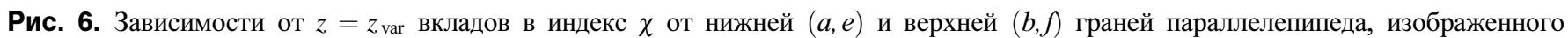
на рис. $1, b$, а также суммы вкладов от его фронтальной и задней граней $(c, g)$ и полной суммы всех вкладов $(d, h)$. Данные соответствуют конфигурациям намагниченности, содержащим две ВБЛ разного типа (случай $B$ на рис. 4 и 5 ), для пленок толщиной $100 \mathrm{~nm}(a-d)$ и $300 \mathrm{~nm}(e-h)$.

на нижней грани

$$
\Phi(0)=\pi+\varphi_{S}, \quad \Phi\left(\frac{1}{4} l_{z}\right)=\pi / 2, \quad \Phi\left(\frac{1}{2} l_{z}\right)=-\varphi_{S},
$$

на фронтальной грани

$$
\Psi(0)=-\varphi_{S}, \quad \Psi\left(\frac{1}{2} l_{y}\right)=0, \quad \Psi\left(l_{y}\right)=\varphi_{S},
$$

на тыльной грани

$$
\Psi(0)=\pi+\varphi_{S}, \quad \Psi\left(\frac{1}{2} l_{y}\right)=\pi, \quad \Psi\left(l_{y}\right)=\pi-\varphi_{S} .
$$

Благодаря тому, что функции (5), (6) были выбраны в факторизованной форме, интегралы (4) для отдельных граней параллелепипеда $G$ могут быть вычислены точно. Их значения зависят только от граничных условий и равны

$$
\chi_{t}=-\frac{1}{2}+\frac{\varphi_{S}}{\pi}, \quad \chi_{b}=\frac{1}{2}+\frac{\varphi_{S}}{\pi}, \quad \chi_{f}=\chi_{r}=-\frac{\varphi_{S}}{\pi} .
$$

Легко видеть, что $\chi_{t}+\chi_{b}+\chi_{f}+\chi_{r}=0$; это условие должно быть выполнено, поскольку внутри образца нет СТ. В случае $\varphi_{S}=0$ ДГ становится блоховской, вихрь в поперечном сечении ДГ исчезает и $\chi_{f}=\chi_{r}=0$, на оси ДГ в области ВБЛ намагниченность поворачивается на каждой из границ пленки на угол $\pi$. В случае $\varphi_{S}=\pi / 2$ в области ВБЛ намагниченность поворачивается на нижней границе на угол $2 \pi\left(\chi_{b}=1\right)$, на верхней границе не поворачивается $\left(\chi_{t}=0\right)$. Аналогичным образом для ВБЛ типа I можно найти

$$
\chi_{t}=\chi_{b}=-\frac{1}{2}+\frac{\varphi_{S}}{\pi}, \quad \chi_{f}=\chi_{r}=-\frac{\varphi_{S}}{\pi},
$$

теперь $\chi_{t}+\chi_{b}+\chi_{f}+\chi_{r}=-1$, так как ВБЛ содержит СТ.

Горизонтальные штриховые линии, наложенные на графики на рис. $6, a, b, e, f$, соответствуют значениям индекса, полученным с помощью формул (8), в которые подставлено численно найденное значение $\varphi_{S}$. Мы видим, что результаты, полученные с помощью модельных функций (5), (6), хорошо согласуются с найденными численно.

\section{4. Динамические перестройки структуры ДГ}

Наблюдая за динамическими изменениями распределения намагниченности в сечениях ДГ различными плоскостями $z=$ const, можно фиксировать моменты рождения и аннигиляции пар вихрь-антивихрь визуально. Чтобы построить графики траекторий центров вихревых структур, воспользуемся приемом, предложенным в работе [19]. Рассчитаем значения числа вращения $\gamma=\int d \Phi(\mathbf{r})$, равного полному углу поворота вектора, принадлежащего некоторому векторному полю, при обходе вдоль определенного замкнутого контура в координатном пространстве. Будем проводить интегрирование по замкнутым прямоугольным контурам $\Gamma$, лежащим на различных плоскостях $z=z_{\text {var }}$ и имеющим 

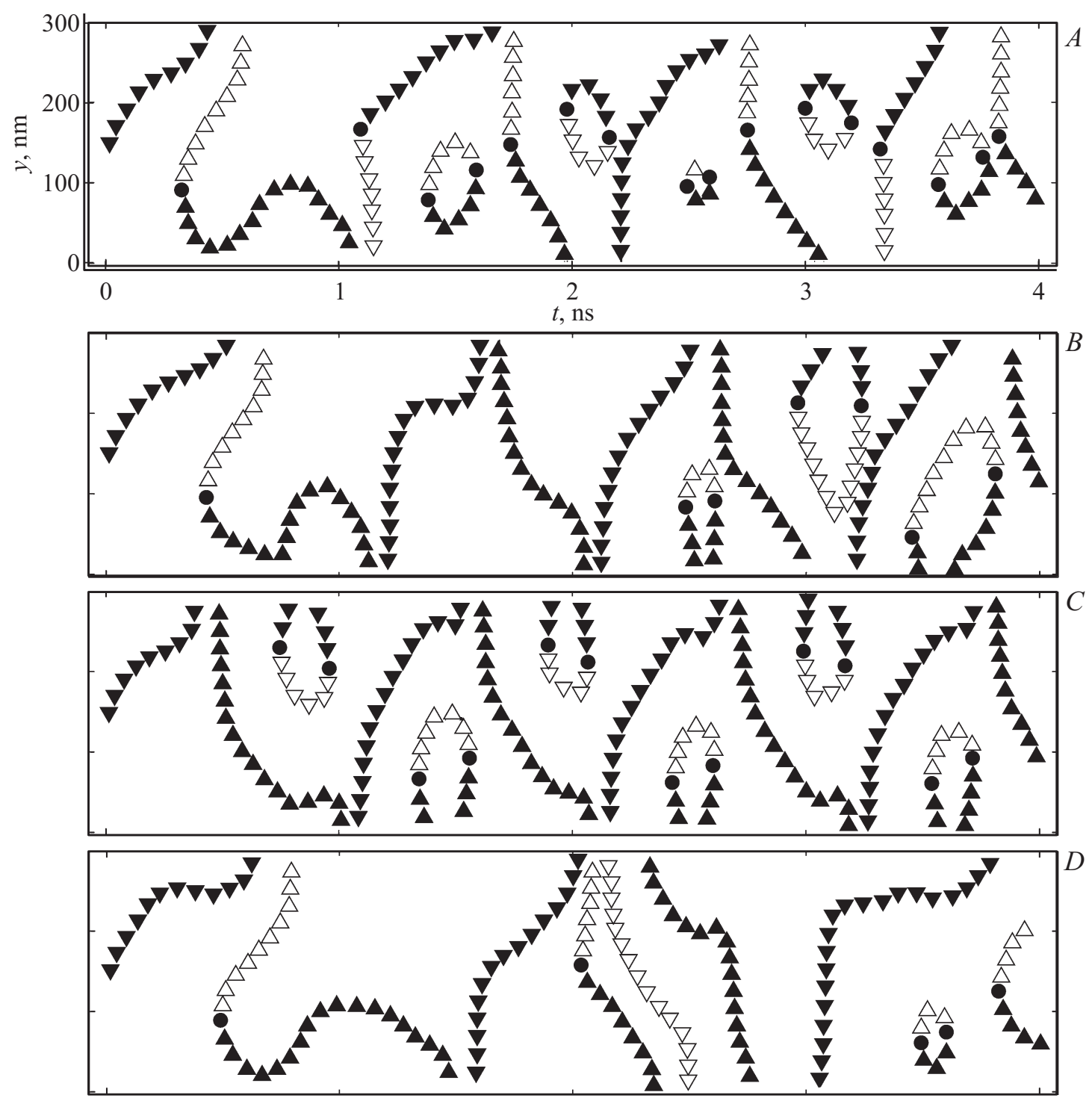

Рис. 7. Зависимости от времени $y$-координат центров вихрей и антивихрей, наблюдаемых в сечении ДГ, содержащей продольные вихревые нити, плоскостью $z=0$ (толщина пленки $300 \mathrm{~nm}$; в качестве начальных условий выбраны конфигурации, изображенные на рис. 5; приложено магнитное поле $H=400 \mathrm{G}$ ). Треугольниками показаны траектории центров вихрей (антивихрей); темным треугольникам соответствуют вихри, светлым - антивихри. Намагниченность в центре вихря (антивихря) направлена вдоль оси $z$ (в противоположном направлении), если горизонтальное основание треугольника находится внизу (вверху). Темными кружками отмечены точки, в которых пары вихрь-антивихрь возникают или исчезают.

различные длины $y_{\text {var }}$ сторон, расположенных вертикально (рис. $1, d)$. В качестве векторного поля возьмем поле нормированных проекций намагниченности на плоскости, ортогональные к оси $z: \tilde{\mathbf{m}}=\mathbf{m}_{\perp} /\left|\mathbf{m}_{\perp}\right|$, где $\mathbf{m}_{\perp}=\mathbf{m}-\mathbf{k}(\mathbf{k m})$ и $\mathbf{k}-$ единичный орт оси $z$. Используем для проведения расчетов формулу [33,34]

$$
\gamma=\frac{1}{2 \pi} \oint_{\Gamma} \mathbf{k}\left[\tilde{\mathbf{m}} \times \frac{d \tilde{\mathbf{m}}}{d \tau}\right] d \tau,
$$

где $\tau$ - параметр, меняющийся при обходе контура Г. Сканируя расчетную область путем выбора различных значений $y_{\text {var }}, z_{\text {var }}$, можно строить различные графические образы вихревых структур.
На рис. 7 изображены зависимости от времени $y$-координат центров вихрей и антивихрей (последние формируются в нашем случае только в динамике) в сечении $z=0$. Мы видим, что вихри и антивихри появляются (исчезают) парами внутри пленки или же поодиночке на граничных поверхностях. Вследствие того, что различия в значениях $x$-координат центров вихрей/антивихрей малы и сопоставимы с погрешностью их нахождения, не меньшей величины шага сетки, график зависимости $x$-координат от времени, аналогичный изображенному на рис. 7, оказывается малоинформативным; мы его опускаем. 


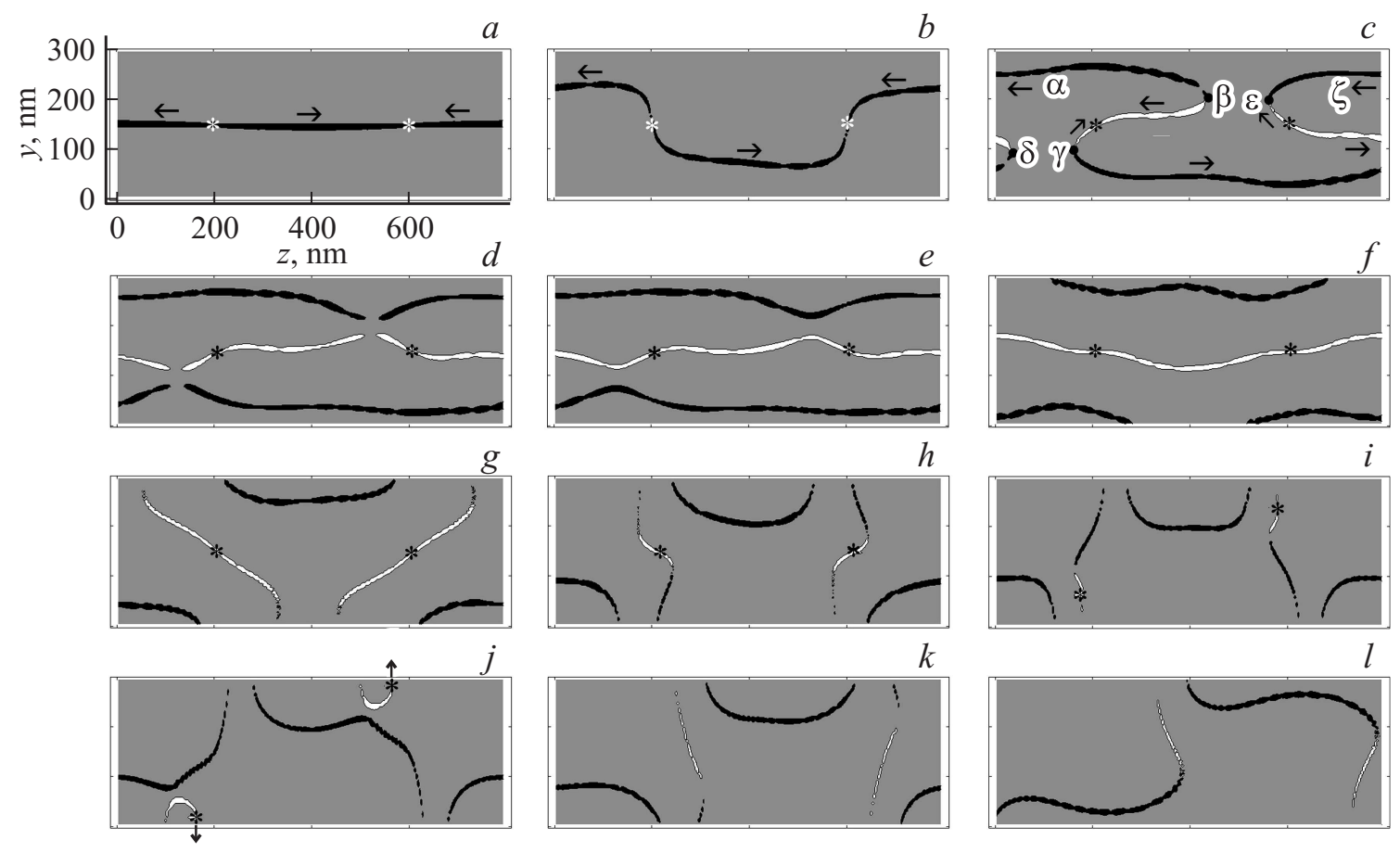

Рис. 8. Проекции вихревых (антивихревых) нитей на плоскость, перпендикулярную границам пленки и параллельную ДГ, для моментов времени $t=0.015(a), 0.18(b), 0.35(c), 0.375(d), 0.385(e), 0.46(f), 0.6(g), 0.7(h), 0.8(i), 0.83(j), 0.91(k)$ и $1.05 \mathrm{~ns}(l)$. В качестве начальной выбрана статическая конфигурация ДГ с двумя ВБЛ, содержащими СТ, в пленке толщиной $300 \mathrm{~nm}$ (случай $A$ на рис. 5); приложено магнитное поле $H=400 \mathrm{G}$.
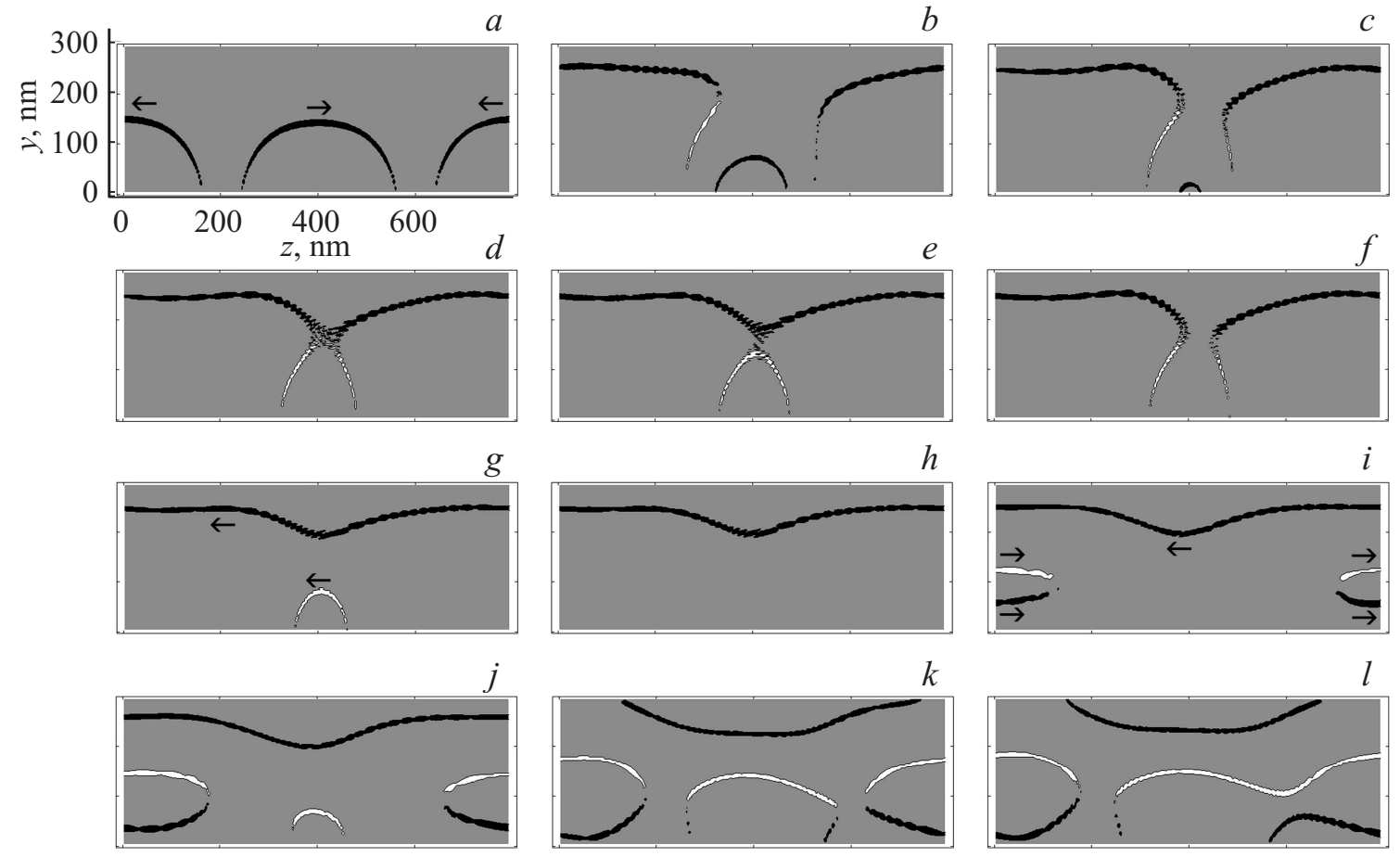

Рис. 9. Проекции вихревых (антивихревых) нитей на плоскость, перпендикулярную границам пленки и параллельную ДГ, для моментов времени $t=0.015(a), 0.3(b), 0.4(c), 0.415(d), 0.425(e), 0.435(f), 0.46(g), 0.48(h), 0.52(i), 0.57(j), 0.66(k)$ и $0.68 \mathrm{~ns}(l)$. В качестве начальной выбрана статическая конфигурация ДГ с двумя ВБЛ без СТ, в пленке толщиной $300 \mathrm{~nm}$ (случай $D$ на рис. 5); приложено магнитное поле $H=400 \mathrm{G}$. 
Рассчитывая индексы $\chi$ путем взятия интегралов (4) по замкнутым поверхностям, изображенным на рис. $1, b$, мы можем находить $z$-координаты СТ как значения $z_{\mathrm{var}}$, при которых $\chi$ меняется скачком на \pm 1 . Аналогичным образом, с помощью другого семейства поверхностей (рис. $1, c)$ можно находить $y$-координаты СТ. Используя этот прием, строим изображения проекций СТ на плоскость $y 0 z$, далее совмещая их с изображениями нитей.

Рис. 8 содержит коллекцию „мгновенных снимков“ проекций вихревых нитей и СТ на плоскость $y 0 z$. Стрелки показывают направления векторов намагниченности в корах вихревых (темные линии) и антивихревых (светлые линии) нитей; звездочками обозначены положения СТ (их координаты найдены описанным выше способом). Было выбрано начальное распределение, изображенное на рис. $5 A$. На рис. $8, a$ видно, что в таком распределении каждая СТ находится на границе двух вихревых нитей с одинаковыми направлениями вращения и встречными направлениями векторов намагниченности в корах. После включения внешнего поля с течением времени $(c)$ появляются $S$-образные структуры, состоящие из чередующихся участков вихревых $([\alpha, \beta],[\gamma, \delta],[\varepsilon, \xi])$ и антивихревых $([\beta, \gamma],[\delta, \varepsilon])$ нитей; при этом СТ оказываются на участках нитей с поперечной структурой антивихревого типа. В дальнейшем (графики $c, d, e)$ точка $\beta$ сближается и сливается с точкой $\varepsilon$, тоже происходит с точками $\delta$ и $\gamma$. В результате образуются нити, идущие вдоль ДГ - две вихревых и одна антивихревая $(d)$. При этом вихревые нити имеют противоположные направления $\mathbf{m}$ в корах, а антивихревая нить расположена между ними по всей длине ДГ. Разрыв антивихревой нити приводит к появлению новых $S$-образных структур, оканчивающихся нитями вихревого типа, выходящими на границы пленки (графики $g, h, i)$. В итоге $(j) \mathrm{CT}$, получив возможность выйти на поверхность пленки, исчезают.

Графики на рис. 9 отвечают случаю движения ДГ при выборе распределения $\mathbf{m}$ с двумя ВБЛ без СТ в качестве начального (рис. 5D). Здесь, как и выше, мы видим вихревые и антивихревые нити, сходящиеся парами во внутренних точках пленки и выходящие на граничные поверхности. На графиках $(i, j, k)$ представлен процесс рождения петли, состоящей из отрезков нитей различного типа, не выходящих на поверхность. Далее $($ графики $j, k)$ появляется еще одна петля с двумя выходами на нижнюю границу пленки.

Наблюдая движение вихревых нитей в течение длительного времени, можно заметить, что в некоторых случаях формируется устойчивая структура, движущаяся с постоянной скоростью вдоль ДГ. Данный результат согласуется с ранее проведенными расчетами [30,31].

\section{5. Заключение}

Динамические процессы в ферромагнетиках представляют собой сложные последовательности структурных перестроек, сопровождающихся возникновением и исчезновением топологических солитонов. Изучение этих процессов позволяет прийти к пониманию физических механизмов, несущих ответственность за макроскопические свойства магнитных сред. Так, генерация спиновых волн, сопровождающая рождение и уничтожение вихревых нитей и СТ в магнитных пленках, вносит свой вклад в диссипацию. С точки зрения перспективной электроники, особый интерес представляет разработка методов стабилизации топологических структур - с целью их использования в устройствах памяти и логических элементах.

Трудности экспериментального изучения быстрой динамики наноразмерных структур склоняют чашу весов в пользу широкого применения методов численного моделирования. Нам кажется важным, используя эти методы, классифицировать типичные сценарии регулярной и нерегулярной динамики намагниченности в ферромагнетиках различного типа, рассматривая их с позиций общей теории нелинейных динамических систем.

Выделим среди результатов, полученных в работе, наиболее важные.

1. В пленках с перпендикулярной анизотропией, при различных значениях их толщины, с высокой точностью рассчитаны трехмерные распределения намагниченности в однородных ДГ и в ДГ с ВБЛ. Установлено, что при уменьшении толщины пленки существенно изменяется распределение $\mathbf{m}$ на ее поверхности в области локализации ВБЛ (указанное относится к ВБЛ, не содержащей СТ). Как следствие, ВБЛ данного типа, ясно различимые только на одной граничной поверхности толстой пленки, становятся хорошо видимыми на обеих поверхностях в пленке меньшей толщины (конкретные значения зависят от параметров материала).

2. Показано, что в пленках рассматриваемого типа различные локализованные структуры могут быть охарактеризованы определенными значениями гомотопического индекса $\chi$. Отличие от случая пленок с плоскостной анизотропией, рассмотренного ранее [20], состоит в том, что эти значения могут быть не кратны \pm 1 или $\pm 1 / 2$, плавно меняясь с изменением толщины пленки.

3. Показано, что статические и динамические распределения $\mathbf{m}$ в ДГ в пленках с перпендикулярной анизотропией могут быть описаны с помощью представления о „вихревых нитях“, предложенного в [20]. Найден способ визуализации движения вихревых нитей и СТ в ДГ, движущейся в постоянном магнитном поле.

4. Установлено, что СТ располагаются на вихревых нитях, выполняя роль элементов, разделяющих участки нитей (как вихревого, так и антивихревого типа) с противоположно направленными намагниченностями в корах. Описаны некоторые сценарии динамических перестроек вихревых структур.

Авторы благодарны А.Б. Борисову, В.В. Киселеву, Ф.Н. Рыбакову за участие в обсуждении работы. 


\section{Список литературы}

[1] С.В. Вонсовский. Магнетизм. Наука, М. (1973). 1032 с.

[2] S.S.P. Parkin, M. Hayashi, L. Thomas. Science 320, 5873, 190 (2008).

[3] M. Hayashi, L. Thomas, R. Moriya, C. Rettner, S.S.P. Parkin. Science 209, 5873, 209 (2008).

[4] D.A. Allwood, G. Xiong, C.C. Faulkner, D. Atkinson, D. Petit, R.P. Corburn. Science 309, 5741, 1688 (2005).

[5] I.C. Slonczewski. JMMM 159, L1 (1996).

[6] D.D. Awschalom, M.E. Flatte. Nature Phys. 3, 153 (2007).

[7] A.R. Rocha, V.M. Garsia-Suares, S.W. Bailey, C.J. Lambert, J. Ferrer, S. Sanvito. Nature Mater. 4, 335 (2005).

[8] A.B. Hamida, S. Sievers, F. Bergmann, A.-M. Racu, M. Bielder, K. Pierz, H.W. Schuacher. Phys. Status Solidi B. 251, 1652 (2014).

[9] L. Landau, E. Lifshitz. Phys. Zeitsch. Sow. 8, 153 (1935).

[10] A.E. La Bonte. J. Appl. Phys. 40, 2450 (1969).

[11] C.C. Shir. J. Appl. Phys. 49, 3413 (1978).

[12] А. Малоземов, Дж. Слонзуски. Доменные стенки с цилиндрическими магнитными доменами. Мир, М. (1982). 384 с.

[13] Б.Н. Филиппов, А.П. Танкеев. Динамические эффекты в ферромагнетиках с доменной структурой. Наука, М. (1987). 216 c.

[14] В.В. Волков, В.А. Боков. ФТТ 50, 193 (2008).

[15] S. Huo, J.E.L. Bishop, J.W. Tucker, W.M. Rainforth, H.A. Davies. JMMM 177, 229 (1998).

[16] S. Huo, J.E.L. Bishop, J.W. Tucker, W.M. Rainforth, H.A. Davies. JMMM 218, 103 (2000).

[17] M. Redjdal, A. Kakay, T. Trunk, M.F. Ruane, F.B. Humphrey. J. Appl. Phys. 89, 7609, (2001).

[18] В.В. Зверев, Б.Н. Филиппов. ЖЭТФ 144, 126 (2013).

[19] В.В. Зверев, Б.Н. Филиппов, М.Н. Дубовик. ФТТ 56, 1725 (2014).

[20] В.В. Зверев, Б.Н. Филиппов. ФТТ, 58, 473 (2016).

[21] J.-P. Adam, N. Vernier, J. Ferré, A. Thiaville, V. Jeudy, A. Lemaitre, L. Thevenard, G. Faini. Phys. Rev. B 80, 193204 (2009).

[22] У.Ф. Браун. Микромагнетизм. Мир, М. (1979). 160 с.

[23] A. Vansteenkiste, J. Leliaert, M. Dvornik, F. Garcia-Sanchez, B. Van Waeyenberge. AIP Adv. 4, 107133 (2014).

[24] F.H. de Leeuw, R. van den Doel, U. Enz. Rep. Prog. Phys. 43, 690 (1980).

[25] A. Thiaville, J.M. Garsia, R. Dittrich, J. Miltat, T. Schrelf. Phys. Rev. B 67, 094410 (2003).

[26] Л.И. Антонов, С.Г. Осипов, М.М. Хапаев. ФММ 55, 917 (1983).

[27] Y. Nakatani, N. Hayashi. IEEE Trans. Mag. 24, 3039 (1988).

[28] Y. Nakatani, N. Hayashi. IEEE Trans. Mag. 29, 2587 (1993).

[29] A. Bagnérés, G.N. Patterson, F.B. Humphrey. IEEE Trans. Mag. 27, 5501 (1991).

[30] A. Bagnérés, F.B. Humphrey. IEEE Trans. Mag. 28, 2344 (1992).

[31] M. Redjdal, P.W. Gross, F.B. Humphrey. IEEE Trans. Mag. 33, 3999 (1997).

[32] A. Bagnérés. IEEE Trans. Mag. 35, 4318 (1999).
[33] Б.А. Дубровин, С.П. Новиков, А.Т. Фоменко. Современная геометрия. Наука, М. (1979), 760 с.

[34] Г.Е. Воловик, В.П. Минеев. ЖЭТФ 72, 2256 (1977).

[35] А.М. Косевич, Б.А. Иванов, А.С. Ковалев. Нелинейные волны намагниченности. Динамические и топологические солитоны. Наук. думка, Киев (1983). 192 с.

[36] А.А. Белавин, А.М. Поляков. Письма в ЖЭТФ 22, 503 (1975).

[37] O.A. Tretiakov, O. Tchernyshyov. Phys. Rev. B 75, 012408 (2007).

[38] S. Komineas. Phys. Rev. Lett. 99, 117202 (2007). 